\title{
Leaping shampoo glides on a lubricating air layer
}

\author{
S. Lee, ${ }^{1}$ E. Q. Li, ${ }^{2}$ J. O. Marston, ${ }^{2}$ A. Bonito, ${ }^{1}$ and S. T. Thoroddsen ${ }^{2}$ \\ ${ }^{1}$ Department of Mathematics, Texas A\&M University, College Station, Texas 77843-3368, USA \\ ${ }^{2}$ Division of Physical Sciences and Engineering \& Clean Combustion Research Center, King Abdullah University of Science and Technology, \\ Thuwal, 23955-6900, Saudi Arabia
}

(Received 4 March 2013; published 10 June 2013)

\begin{abstract}
When a stream of shampoo is fed onto a pool in one's hand, a jet can leap sideways or rebound from the liquid surface in an intriguing phenomenon known as the Kaye effect. Earlier studies have debated whether non-Newtonian effects are the underlying cause of this phenomenon, making the jet glide on top of a shear-thinning liquid layer, or whether an entrained air layer is responsible. Herein we show unambiguously that the jet slides on a lubricating air layer. We identify this layer by looking through the pool liquid and observing its rupture into fine bubbles. The resulting microbubble sizes suggest this air layer is of submicron thickness. This thickness estimate is also supported by the tangential deceleration of the jet during the rebounding.
\end{abstract}

PACS number(s): 47.50.- d

The fascinating phenomenon of a leaping shampoo stream was first studied half a century ago by Kaye [1], followed by Collyer and Fisher [2]. More recent advances are due to Versluis et al. [3], who managed to produce stable leaping by directing the jet onto an angled pool, showing up to five repeated leaps. They characterize the leaping behavior and suggest that non-Newtonian effects are crucial for the leaping, proposing the presence of a $100 \mu \mathrm{m}$-thick layer of a shearthinning liquid between the jet and the pool.

However, the presence of an air layer has already been shown to cause leaping of Newtonian jets by Swinney and coworkers [4]. Binder and Landig [5] have also given indirect evidence for the presence of an air layer by shining a light along the jet and showing that it acts as a light guide. For this to occur, the authors postulate an air layer must be present to produce the very large index of refraction differences needed. The most recent experiments also suggest the presence of an air layer [6], but without direct observations. Despite the above indications the presence of an air layer for the highly nonNewtonian shampoo has not been conclusively decided up until now $[7,8]$.

Herein we show direct visual evidence that during the leaping there is indeed always a lubricating layer of air between the jet and pool surfaces.

Figure 1 shows a typical leaping shampoo jet along with a definition sketch. The jet is gravity fed through a hole in a large container, kept at a constant height above a pool of the same liquid. The hole is typically $1-3 \mathrm{~mm}$ in diameter, and the corresponding minimum fall heights required for leaping are from 25 to $40 \mathrm{~cm}$.

The liquid used in the presented experiments was a commercial shampoo [9]; see the Supplemental Material for the main ingredients [10]. Its shear-thinning properties are best characterized with the Carreau model, as shown in Fig. 2. This is non-Newtonian behavior similar to that presented by Versluis et al. [3]. The surface tension of the shampoo is $\sigma=$ $30 \mathrm{mN} / \mathrm{m}$. We have tested various other shampoo brands, obtaining qualitatively similar air entrainment results.

Following Versluis et al. [3], we stabilize the direction of the rebounding jet by inclining the pool, by about $14^{\circ}$. This allows high magnification in-focus imaging within the pool.
The large viscosity of the shampoo allows only slow downhill gliding of the pool, at a pool surface velocity of about $4 \mathrm{~mm} / \mathrm{s}$. We confine the pool to a narrow channel between two glass walls, through which we can view the jet-turning region within the pool.

The rapid breakup of the thin air layer requires high-speed video imaging at frame rates up to 12000 frames/s, which we acquired with a Phantom V1610 CMOS video camera. We use a long-distance microscope (Leica Z16 APO) at optical magnifications up to $4 \times$, giving $7 \mu \mathrm{m} /$ pixel. Backlighting was accomplished with a $350-\mathrm{W}$ metal-halide lamp (Sumita), shone onto a diffuser.

Figure 3 shows the overall view of the leaping jet. When the jet initially hits the pool surface, it usually starts coiling, forming a small heap. Subsequently, it starts sliding down along the surface of this heap and exits at a small angle to the horizontal along the pool surface, as shown in the first frame. The vertical force required to turn the jet has an opposite reaction on the pool, pushing downwards to form a dip into the pool surface (second frame). When this dip becomes deeper, it turns the jet by a larger angle, directing it more in the vertical, as it leaves the pool. This proceeds and the jet points progressively more vertically until it can intersect the incoming jet. The jet leaving the pool is significantly thicker than the one entering it, indicating it is decelerated by viscous tangential forces $[3,6]$.
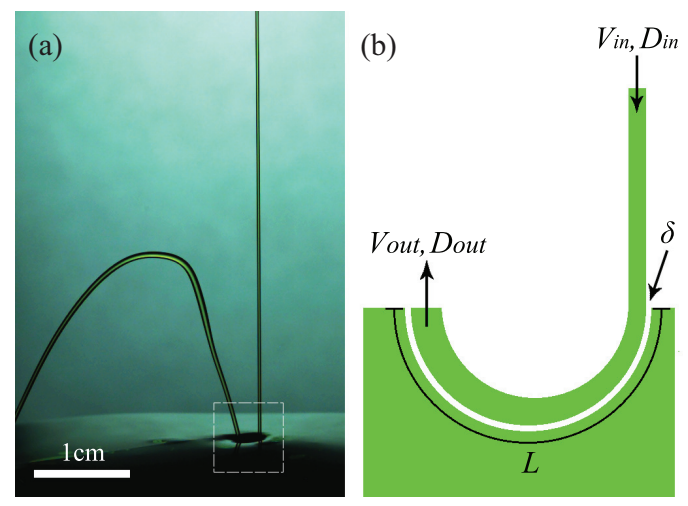

FIG. 1. (Color) (a) Typical leaping shampoo jet and (b) definition sketch. 


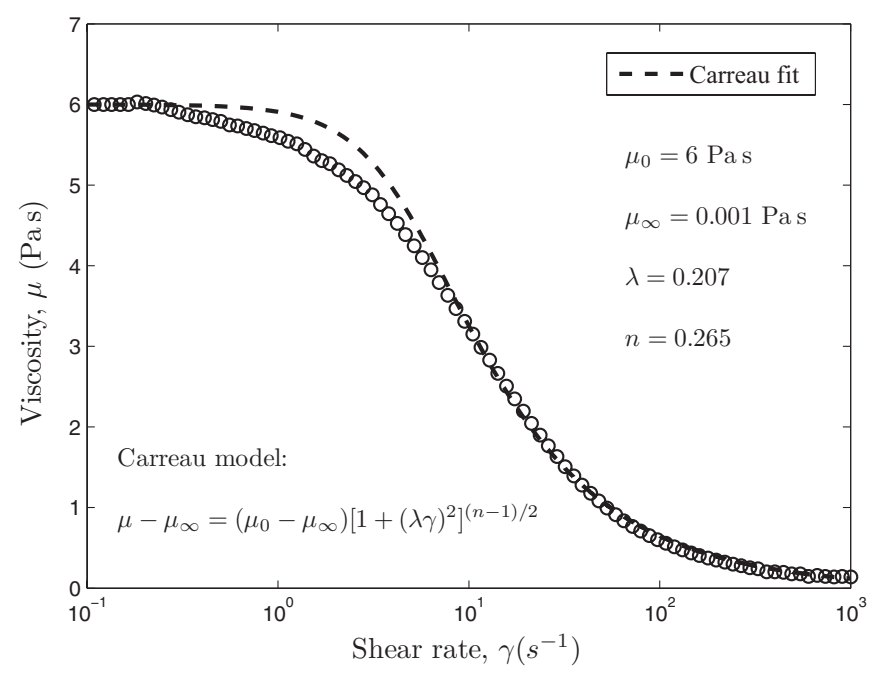

FIG. 2. Shear-thinning properties of the shampoo at $21.5^{\circ} \mathrm{C}$.

Below we will use this deceleration to estimate the thickness of the air layer. The fourth frame shows a sudden disturbance of the jet, associated with a rupture of the air layer, which breaks up in the fifth frame (white arrow). Immediately during this breakup, the outgoing jet starts falling towards the pool, and its diameter thins, as it is no longer fed by the incoming jet. In the last two frames, the jet has again started coiling at the pool surface, before again sliding down the heap to begin a new cycle. Myriad bubbles are visible within the pool from earlier breakups of the air layer.

Figure 4 shows a close-up sequence of frames showing the breakup of the air layer, which occurs over about $40 \mathrm{~ms}$. The layer first breaks up into filaments and then small bubbles. By measuring the spacing and volume of the air threads, we can estimate the thickness of the original air layer $\delta$ by matching the two volumes, i.e., $\pi d^{2} / 4=\delta W$, where $d$ is the thread diameter and $W$ is their spacing, indicated by arrows in the fifth frame. This gives a local air-layer thickness of $\delta \sim 0.54 \mu \mathrm{m}$.

Some of the video sequences show both the incoming and outgoing jet as well as the size of the turning region within the pool, like the one shown in Fig. 3. By seeding the shampoo with small particles we can track them to quantify the velocity in the different regions. The incoming jet in Fig. 3 is slightly out of focus but still allows us to measure accurately its diameter $D_{\text {in }}$ and velocity $V_{\text {in }}$. This was verified with the liquid continuity $V_{\text {in }} / V_{\text {out }}=\left(D_{\text {out }} / D_{\text {in }}\right)^{2}$, showing closure within $4 \%$ between the incoming and outgoing streams.

The jet is decelerated by the viscous stress at its free surface $[3,6]$. The amount of deceleration lets us estimate the strength of this viscous stress, which in turn gives us the thickness $\delta$ of the air layer under the jet. A similar friction model was used by Ochoa et al. [6], who managed to predict the minimal incoming jet speed required to start the Kaye effect. By constructing a steady control volume coinciding with the air film, we can estimate the total tangential shear force along the bottom of the jet:

$$
F_{\text {tang }}=\mu_{\text {air }} \frac{\Delta V}{\delta} S=\rho_{\text {jet }}\left(V_{\text {in }}^{2} A_{\text {in }}-V_{\text {out }}^{2} A_{\text {out }}\right),
$$

where $\mu_{\text {air }}$ is the viscosity of air, $\Delta V$ is the velocity jump across the air film, $S$ is the integrated area of the jet which is riding on the thin air film, $\rho_{\text {jet }}$ is the liquid density, and the $A$ 's are the local cross-sectional areas of the jet, entering and leaving the control volume. We estimate $S$ by $\pi\left(D_{\text {in }}+D_{\text {out }}\right) L / 4$, where $D_{\text {in }}, D_{\text {out }}$, and $L$ are the incoming and outgoing jet diameters and the interaction length of the flowing jet with the pool, as defined in Fig. 1. Combining with continuity, we can solve for the air-layer thickness,

$$
\delta=\frac{\mu_{\text {air }}}{\rho_{\text {jet }}}\left(\frac{S}{A_{\text {in }}}\right) \frac{\Delta V}{V_{\text {in }}^{2}-V_{\text {out }} V_{\text {in }}}=0.50 \mu \mathrm{m},
$$
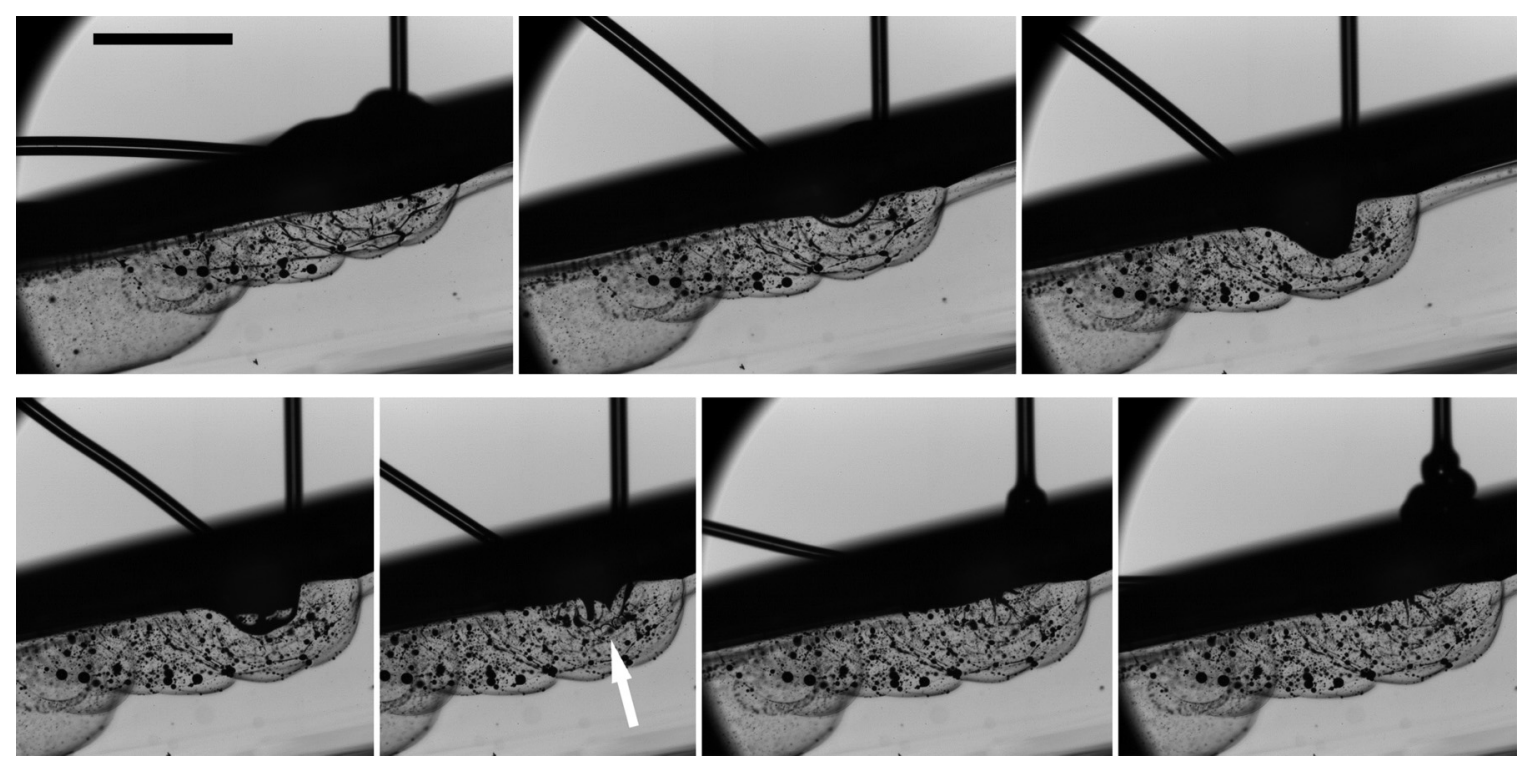

FIG. 3. Overall view of the shampoo jet bouncing off an inclined pool of the same liquid. The scale bar is $3 \mathrm{~mm}$ long. The white arrow points at the breakup of the air layer into threads and bubbles. The relative times of the frames are $t=0,124,287,296,308,324$, and 335 ms. The incoming jet is $0.30 \mathrm{~mm}$ in diameter, approaching the pool at $1.20 \mathrm{~m} / \mathrm{s}$. See also the video in the Supplemental Material [10]. 

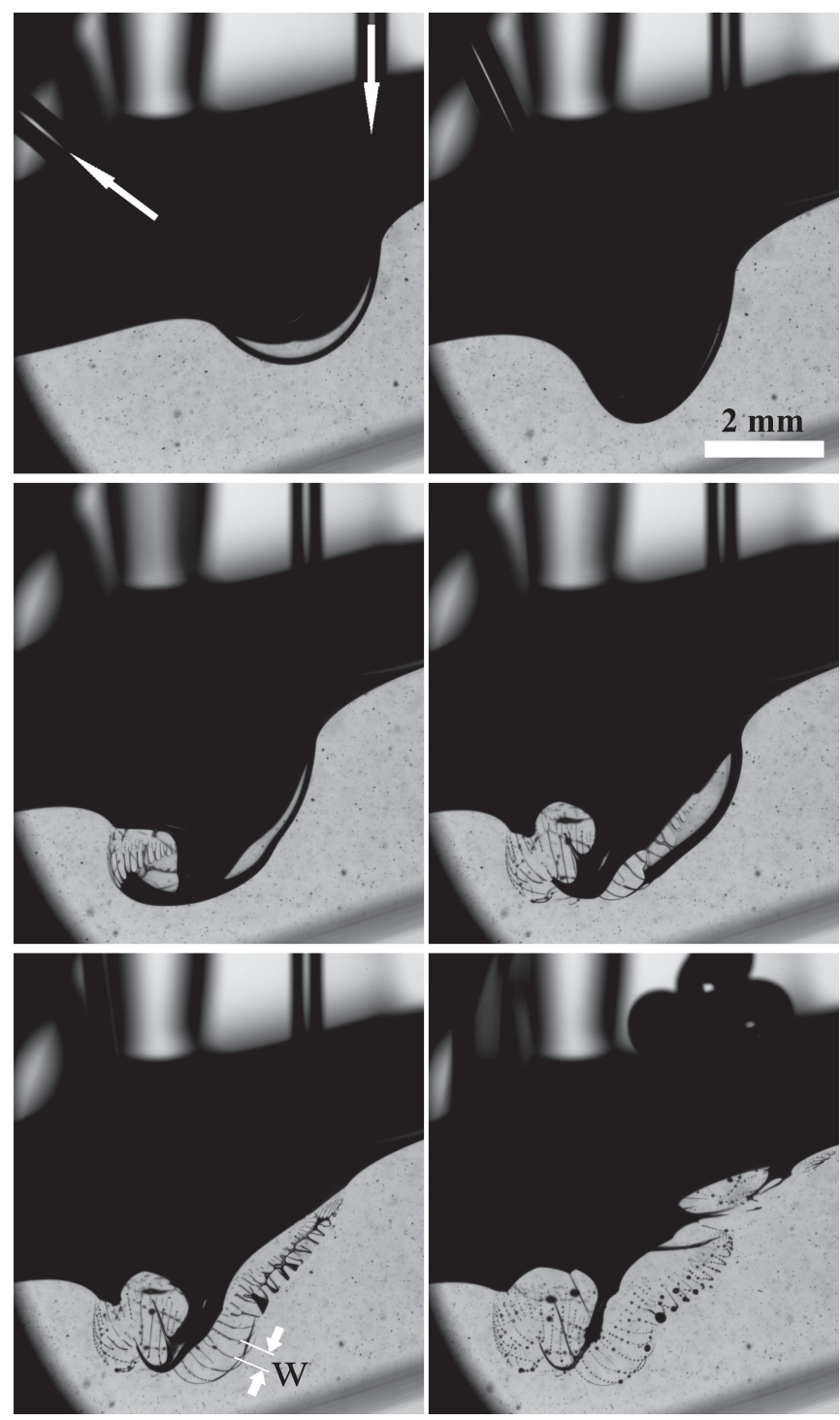

FIG. 4. Breakup of the air layer under the jet. Times relative to first observed rupture are $t=-717,-189,20,31,40$, and $132 \mathrm{~ms}$. The incoming jet is $0.56 \mathrm{~mm}$ in diameter and approaches the pool at $1.59 \mathrm{~m} / \mathrm{s}$. See also the video in the Supplemental Material [10].

which is of similar thickness to that evaluated from the microbubble sizes in Fig. 4. Keep in mind that the estimate of $S$ is quite uncertain and the air thickness will vary along the periphery of the jet, where the lubrication pressure will tend to push the air from the bottom up along the sides. The Reynolds number of the air-flow within the micron-size air layer is of the order $\operatorname{Re}=\delta \rho_{\text {air }} \Delta V / \mu_{\text {air }} \sim 0.1$, verifying laminar flow used in this model.

By seeding small particles into the jet liquid we can measure the tangential velocity within the section of the jet moving below the original pool level. Keep in mind that we can only visualize within a fraction of the jet diameter, owing to the strong diffraction at the liquid-air interfaces. Figure 5 shows trajectories of a few particles, which we use to calculate the local velocities. There is no clear transverse velocity gradient within this section of the jet, down to about $50 \mu \mathrm{m}$ from the bottom. The velocity is even slightly faster closer to the bottom
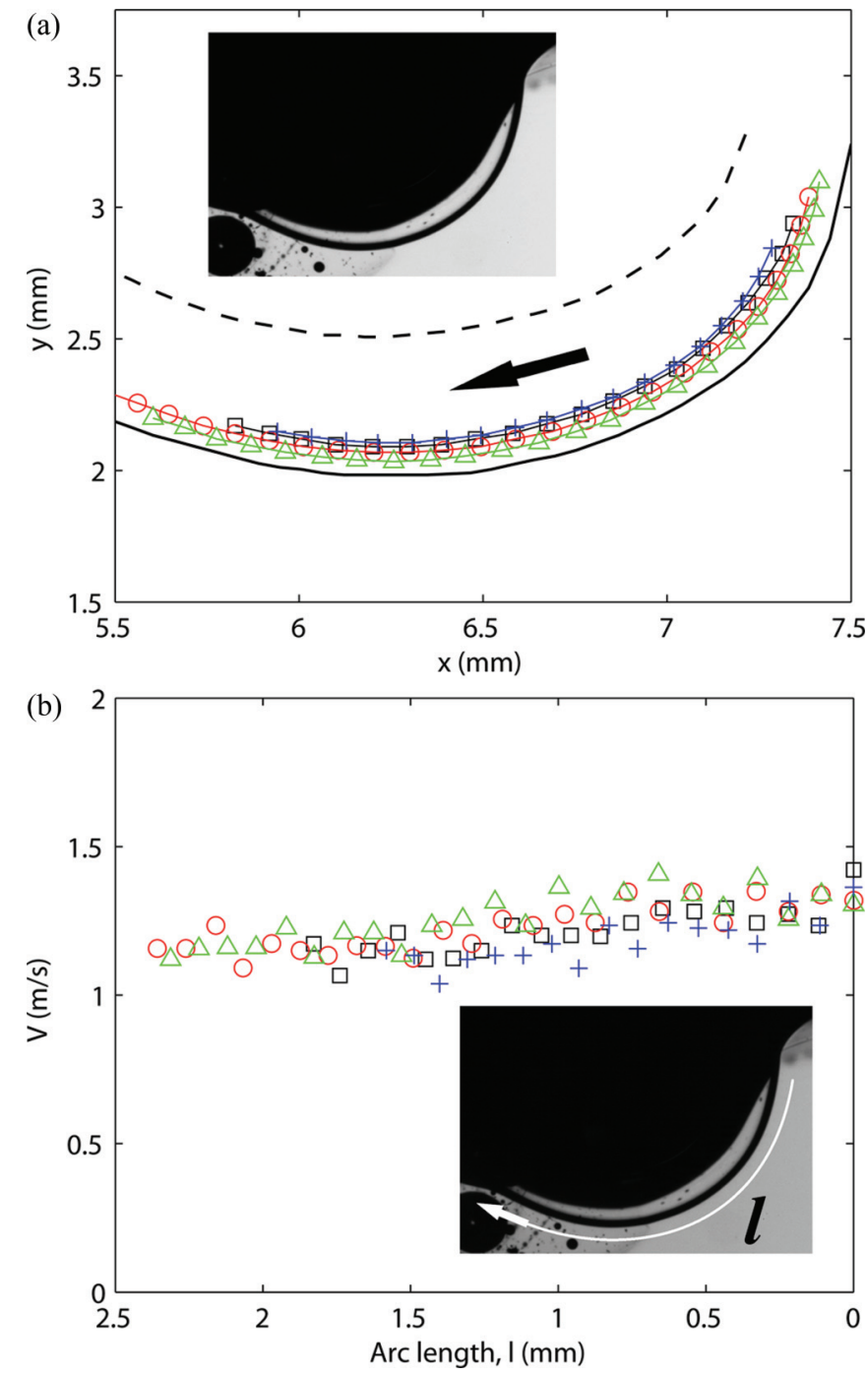

FIG. 5. (Color) (a) The trajectory of typical particles within the jet. The solid curve marks the air layer, and the dashed curve indicates the upper side of the jet if the cross section remains circular. (b) The velocity vs horizontal location along the curving jet.

by geometric effects. When seeding particles into the pool, the velocity under the air layer is insignificant, always less than $4 \mathrm{~mm} / \mathrm{s}$. This supports our assertion that the shear is mostly confined within the thin air layer and rules out a $100-\mu \mathrm{m}$ shear-thinning layer substituting for the air film [3]. On the other hand, Fig. 5(b) shows that the tangential velocity clearly reduces along the jet due to the underlying shear stress at the boundary.

The sharp diffraction of the light at the lower boundaries of the jet in Figs. 3 and 4 demands the presence of an air layer [5]. Even if the refractive index were to change slightly in the earlier-proposed shear-thinning layer, it would not leave "index-of-refraction" bubbles once the shearing stopped.

Similar stable submicron-thin layers of air occur, for example, during the rebounding of a drop impacting onto a pool surface, where the thin film only breaks when it reaches the order of 100-nm thickness (see [11]). The presence of the surfactants may further stabilize this air layer. It remains to be studied how the surfactants interact with the strong shear in 
the air layer and whether their presence stabilizes this layer, as in the soap film case [12]. For surfactants in water, a thin air layer shows irreproducible breakup [13].

We thereby propose that the Kaye effect has more to do with the stability of the air layer rather than the non-Newtonian behavior inside the jet. However, there is no evidence yet of leaping for Newtonian jets coiling onto stationary pools, as Thrasher et al. [4] needed rapidly moving pools to observe leaping of Newtonian liquids. Therefore, non-Newtonian effects could play a role during the initial formation of the air layer or help sustain the air layer.

S.L. and A.B. are partially supported by Award No. KUSC1-016-04, made by King Abdullah University of Science and Technology. We thank Sahraoui Chaieb for the use of his rheometer.
[1] A. Kaye, Nature (London) 197, 1001 (1963).

[2] A. A. Collyer and P. J. Fisher, Nature (London) 261, 682 (1976).

[3] M. Versluis, C. Blom, D. van der Meer, K. van der Weele, and D. Lohse, J. Stat. Mech. (2006) P07007.

[4] M. Thrasher, S. Jung, Y. K. Pang, C.-P. Chuu, and H. L. Swinney, Phys. Rev. E 76, 056319 (2007).

[5] J. M. Binder and A. J. Landig, Eur. J. Phys. 30, S115 (2009).

[6] J. E. Ochoa, C. R. Guerra, and C. Stern, in Experimental and Theoretical Advances in Fluid Dynamics, edited by J. Klapp et al. (Springer, Berlin, 2012), pp. 419-427.
[7] N. M. Ribe, M. Habibi, and D. Bonn, Annu. Rev. Fluid Mech. 44, 249 (2012).

[8] M. Versluis, Exp. Fluids 54, 1458 (2013).

[9] Farmasi baby care, http://www.farmasi.com.tr.

[10] See Supplemental Material at http://link.aps.org/supplemental/ 10.1103/PhysRevE.87.061001 for main ingredients.

[11] S. T. Thoroddsen, M.-J. Thoraval, K. Takehara, and T. G. Etoh, J. Fluid Mech. 708, 469 (2012).

[12] G. Kirstetter, C. Raufaste, and F. Celestini, Phys. Rev. E 86, 036303 (2012).

[13] B. H. Mills, J. R. Saylor, and F. Y. Testik, AIChE J. 58, 46 (2012). 